\title{
International Master Program on Mechatronic Systems for Rehabilitation
}

\author{
Véronique Perdereau ${ }^{1}$, Giovanni Legnani ${ }^{2}$, Vivianne Pasqui $^{1}$, Emilio Sardini $^{3}$, Antonio Visioli $^{3}$ \\ ${ }^{1}$ Université Pierre et Marie Curie - Paris 6, Master Sciences de l'Ingénieur, \\ 4 place Jussieu 75252 Paris cedex 05, France, veronique.perdereau@upmc.fr \\ ${ }^{2}$ Dip. Ing. Meccanica e Industriale, Università di Brescia, \\ Via Branze 38, 25123 Brescia, Italy, Giovanni.Legnani@ing.unibs.it \\ ${ }^{3}$ Dip. Ingegneria dell Informazione, Università di Brescia, \\ Via Branze 38, 25123 Brescia, Italy
}

\begin{abstract}
This paper describes a second level of university course called Master in France, Laurea Magistrale in Italy, jointly organized by UPMC Université Pierre et Marie Curie - Paris (France) and UniBS Università degli Studi di Brescia (Italy). During this "double-degree" course, students coming from these universities share two semesters while studying subjects related to mechatronics and biomechanics. At the end of the program the students obtain a double degree from both universities.
\end{abstract}

Keywords: Assistive robotics, physically interacting robots, sensori-motor functions, multimodal perception, verbal communication, haptics, safety and acceptability

\section{INTRODUCTION}

While the Bologna process aims to harmonize studies in Europe, there is still a clear deficit of outgoing students in all universities. Reasons for such a report are related to material means, visibility and equivalence of studies abroad and probably also fear of unknown. In this context, Université Pierre et Marie Curie (Paris, France) and Università di Brescia (Brescia, Italy) have signed a partnership agreement and jointly propose a turnkey program: negotiated, financed and accompanied so that students entering the program will spend periods of time in a foreign country in a coherent curriculum and receive a double degree from both universities.

The growing importance of health and the aging of the population have opened new applicative fields in the medical or paramedical sectors, especially for diagnosis and rehabilitation. This highlights the necessity to form technicians and engineers that, in cooperation with medical personnel, are able to design and maintain suitable mechatronic devices. This program proposes a specialization in the forefront of the innovation: engineering in the service of the human being (Fig.1).

The adoption of mechatronics or robotized devices for assistance and rehabilitation gives the possibility to improve diagnoses and therapy both in structured places like hospitals or reeducation centers as well as at the patient's own home. With respect to manual therapy, the robotized one is more reproducible, more repeatable, less dependent to the therapist ability, less tiring for the therapist and sometimes may be remotely performed at the patient home. The results are a better quality of life and a reduction of health expenses.

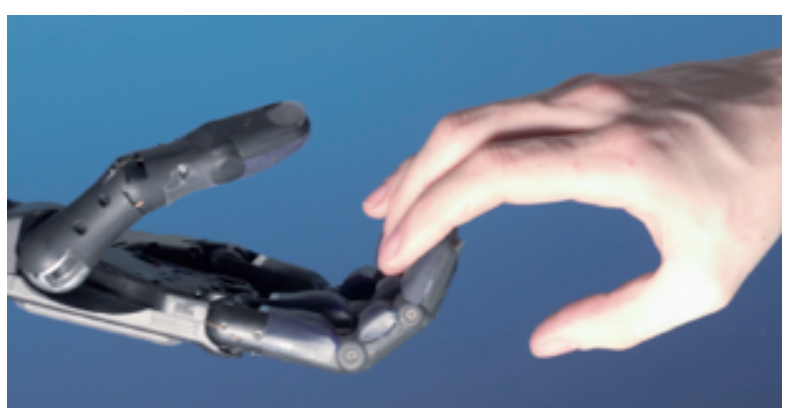

Fig. 1: The Shadow robot hand

\section{PROGRAM DESCRIPTION}

\subsection{Partners}

This program is the result of a fruitful collaboration between two sister universities.

\subsubsection{UPMC}

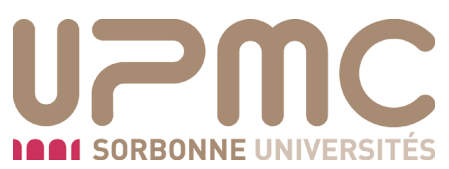

The University Pierre et Marie Curie - Paris 6 (UPMC), devoted to science and medicine, is one of the largest universities in France: 5820 researchers-teachers and clinical teachers, 180 laboratories, 31000 students, 745 scientific PhD defended per year. UPMC is involved in numerous European and international partnership agreements (European research projects, international Masters, etc.). This gives access to facilities only found at the largest institutions: France's largest scientific library, scientific infrastructure facilities, 
large conference venues and strong intellectual environment.

UPMC hosts ISIR (www.isir.fr), a research laboratory which conducts cross-disciplinary research in complex mechanical systems, systems and control, and signal processing. It pursues the application of robotic and intelligent systems to biology, medicine, and neuroscience through fundamental and applied research.

\subsubsection{UNIBS}

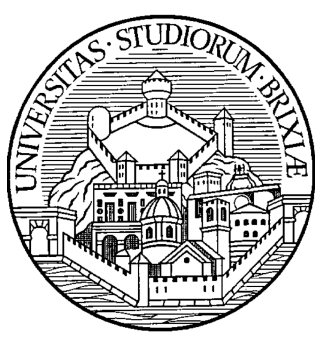

Founded in 1982, the University of Brescia is today one of the largest in Italy with 573 professors and researchers and more than 14,000 students enrolled. The academic activities are conducted within the four faculties of Economics, Engineering, Law and Medicine, which organize a total of 25 three-year degree programs, 17 master degree programs and 4 single cycle degree courses, plus 2 Masters and 41 schools of specialization. The research is conducted by 15 departments which in turn organize $20 \mathrm{PhD}$ programs and 34 research centers.

University of Brescia (UniBS) enters the project with two department units of the engineering faculty: DII (Dept. of Information Engineering, Engineering Faculty) and DIMI (Dept. of Mechanical and Industrial Engineering, Engineering Faculty). Both departments have experience in robotics, mechatronics, rehabilitation devices and have official cooperation with local hospitals.

\subsection{Semester organization}

The project is organized (Fig. 2) in two years corresponding to four semesters (S1, S2, S3, and S4). Students are independently selected by the two partner universities and spend the first semester at their home institution (UPMC or UniBS) where they receive a basic mechatronic education. The first semester syllabus is different in the two universities to take into account the differences in the student background and in national legislation and harmonize student skills for the next semester.

During the second semester the UPMC students join the UniBS's in Brescia where they study the methodologies for modelling mechatronic systems and get a basic of biomechanics. The third semester is spent at UPMC, there the students specialize themselves on mechatronics systems cooperating with human beings for diagnoses, assistance, reeducation and rehabilitation. The fourth semester is dedicated to labs and/or internship and the development of the master thesis that can be performed at UPMC, UniBS or any other public or private body throughout the world. The students presently defend their theses in their home university with a local jury. The possibility of establishing mixed juries with both Italian and French teachers is under discussion.

The first semester is taught in national language, the second semester used to be in Italian since now, but starting from the next year will be in English. The third semester is in English. Students are supplied with educational material in English.

\subsection{Double diploma}

At the end of the course, the students are awarded the title "Master Sciences et Technologies mention Sciences de l'Ingénieur" by UPMC and "Laurea Magistrale in Ingegneria dell'Automazione Industriale" by UniBS. Both degrees give access to doctoral studies or jobs in engineering. In particular, the Italian degree gives access to the habilitation test to start a career as engineer in the field of "Industrial Engineering" or "Information Engineering".

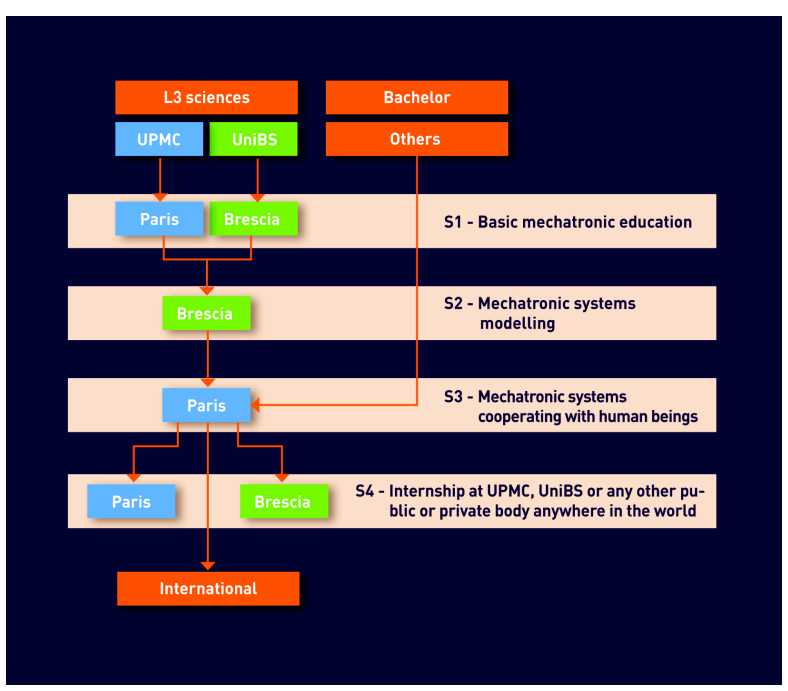

Fig. 2: The scheme of the semester organization

\section{COMPETENCES ACQUIRED}

The first year provides all the mechatronic basics to train engineers capable of handling all kinds of computer-controlled or microprocessor-controlled modern systems.

During the second year, the knowledge is completed by human-machine interaction aspects and understanding of human behavior, useful to the development of mechatronic systems for rehabilitation (diagnosis assistance, functional rehabilitation and assistance).

- Design of mechanical parts taking into account reliability constraints and operational safety

- Integration and control of actuators

- Instrumentation of the platform for the compilation of human-sent signals

- Development of information-processing algorithms (signal or image) for therapeutic purposes 
The full content of the courses is given in appendix.

Part of the courses is taught in English, at UPMC and UniBS. But this training is also a good opportunity for students to learn Italian during the first year or French during the second one.

The opportunities following this curriculum are in companies which design and build mechatronic systems for medical or industrial use. The largest hospitals are already being equipped with the first existing systems and a growing number of hospitals are following this trend. This knowledge background also attracts research given the emergence of this new field of application and the current findings in many domains such as neurophysiology, surgery or physics medicine.

The competences acquired during this program for systems in medical environment are also useful in many other modern life systems as for example sport or leisure equipments, means of transportation, aeronautics, interactive games, robots, tool machines, etc. leading to many other professional opportunities.

The following figures (Fig. 3 and Fig. 4) show two significant examples of student's activity during the program.

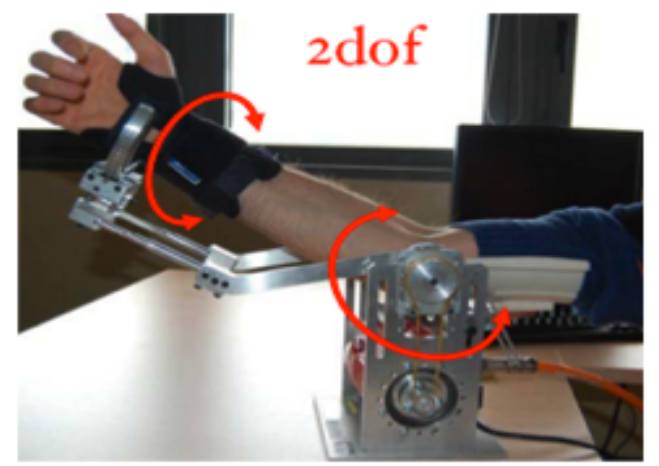

Fig. 3: Mechatronic device for the rehabilitation of the upper limb (flexion extension of the elbow and Pronosupination). The French student Ady Ragou is developing the control of this device at UniBS as project for his Master degree.

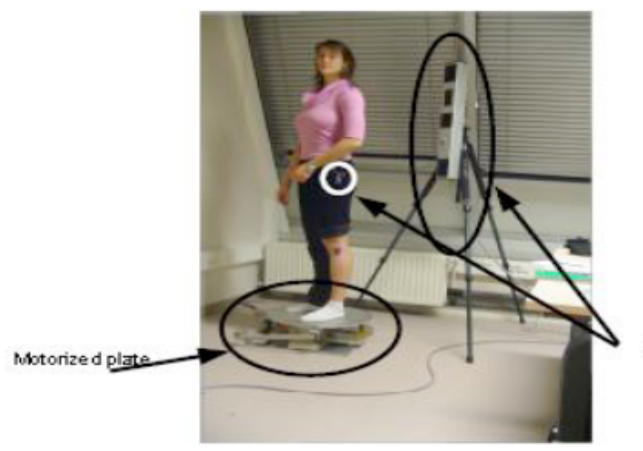

Motion capture syste

Fig. 4: Dynamic platform for postural disturbance. The Italian student Fabio Spazzini has developed the control of this device enslaving its motion to a motion capture system at UPMC as project for his Master degree.

\section{MODALITIES}

\subsection{Promotion}

The promotion of the program is made each year towards the Bachelor students via different means: web site, brochures, oral presentations, advertisement in specific documentation as produced by Campus France.

\subsection{Enrollment}

According to the grants offered, this program of excellence has a limited number of places. Applicants are first selected on their curriculum. The final decision may be taken after an oral evaluation of their motivation, autonomy and level in English.

\subsubsection{Enrollment in first year}

Provided they have the necessary pre-requisites, this curriculum is open to students holding a Bachelor degree in Mechanics, Electronics, Computer science, Physics, Mechatronics. It is also open to medical students who would like to complete their training. At the beginning of the courses, it is not necessary to be fluent in Italian for the French students nor fluent in French for the Italian ones but a level B1 in English is required in both universities.

\subsubsection{Enrollment in second year}

Students may join the second year provided they are engineers or have completed 4 years after highschool graduation, seeking a specialty in research. They can be of all nationalities since courses during the third semester are taught in English.

\subsection{Student mobility}

The students admitted in this program receive a strong help with mobility.

\subsubsection{Financial help}

The students benefit from financial help, about 450 euros/month, to cover the cost of their mobility in Italy during the second semester or in France during the third semester. Normally, students enrolled at UPMC pay 350 euros/year and those enrolled at UniBS pay between 300 euros/year and 2500 euros/year depending on the financial situation of their family. The two establishments have also implemented an exchange agreement, which exempts students from tuition fees in the other university. The cost of the fourth semester internship is often covered by a grant from the hosting structure and can be completed, depending on the destination, by bilateral agreements or the Leonardo program. The program has also received a private grant (Fondazione Cariplo) winning a competitive selection between high level educational proposals. 


\subsubsection{Assistance with organization}

The international relation office of each universities accompanies the students of the program in all practical aspects: enrollment and registration, social service, language courses, life on campus, and integration in the city or any other question which could rise.

\subsubsection{Help for finding accommodation}

UPMC and UniBS help the students in the exchange program by giving them priority access to university accommodations during their mobility.

\subsubsection{Pedagogical monitoring}

The students are supervised during the entire program and even more specifically during their mobility by a pedagogical tutor at their host university.

\subsection{Teacher mobility}

Teachers of the partner universities assure the coordination of the teaching and of the administrative aspects by constant email or phone contact and by regular visits to the partner university. At least one Italian teacher visits UPMC during the first semester of each year while some Italian students are in Paris, and at least one French teacher visits UniBS during the second semester while the French students are in Italy. During their stay in the host university, they participate to some course units. To support the cost of their mobility, teachers receive a financial support like visiting professor positions from both institutions.

\subsection{Course validation}

At the end of the program, the students develop their Master thesis to be defended in one of the two partner universities. The time spent studying at UniBS by the students enrolled at UPMC is recognized by UPMC. The marks awarded in Italy allow the validation of the second semester S2, if necessary through compensation that is by means of the average of all marks, and the delivery of the Master diploma of Engineer Science. The courses attended during the period of mobility clearly appear in the diploma supplement. Similarly the period of study performed at UPMC by students enrolled at UniBS is recognized by UniBS which delivers the diploma "Laurea magistrale in ingegneria dell'automazione industriale", Master in industrial automatisation, compiling the marks obtained at UPMC. In this case all French units must be independently acquired according to the Italian law.

At the end of the program students when successful receive both the Italian and the French degrees.

\section{ANALYSIS OF PAST EXPERIENCE}

The project started in the academic year 2008/2009. About 15 students a year take part in the project ( 5 for each university: UPMC and UniBS and 5 from other foreign universities). This group forms a truly international student group. During the second semester, the students from UPMC are twinned with Italian students from the international program upon their arrival in Brescia. This helps them integrate more smoothly. This Franco-Italian group comes to France for the third semester to study specialized courses and keep working together. Students of other nationalities join them to attend this third semester. The supervision is narrow and teachers are available to give any requested help. To the student and teacher opinion, all this gives a friendly and enriched atmosphere.

The students' mobility is planned during two semesters which enables them not only to benefit from very high-level teaching but also gain linguistic and cultural openness, thus increasing their adaptability. Their character and behavior education take advantage of living one semester abroad in a country with a different culture, to attend courses organized in a different style and using a different language. This mobility experience provides significant added-value when applying for leading foreign labs, and for their recruitment in the emerging companies in this domain, often branches of foreign companies.

The students participating in the project appear very happy of such experience which permits them to study this rather new and challenging subject. To produce systems which help human beings is very motivating, as well as the additional challenge of making robots and humans cooperate.

\section{CONCLUSION}

This joint program is for both universities an excellent basis for deeper cooperation at $\mathrm{PhD}$ level as well as for collaborative research work. They are now looking for an additional partner to extend the program and possibly prepare an Erasmus mundus project. They consider this first experience as essential to be stronger in a larger program.

Students who have attended the program, have definitely shown interest and some of them, both French and Italian, have decided to carry out the Master thesis activity in the other university.

The experience of living abroad and studying in a different university with different organization, but within a coordinated curriculum proved to be very effective for the social, cultural and educational growth of the students. Although it is too early to give a final answer, it is foreseen that their experience will be positively evaluated by the working market. 


\section{Appendix \\ Detailed description of the course units}

This section describes with a few keywords the full content of all the courses taught during this 2-year international Master program.

\section{Semester S1 in Paris}

The courses issued in Paris during the first semester and attained by the French students are:

- Automatic control (3 ECTS)

Mathematical modeling of physical systems. Linearization of non-linear systems. Time domain and frequency domain analysis of system behaviour. Principles and objectives of feedback control. Design of analog controllers.

- Digital signal processing (3 ECTS)

Reminders on sampling, Z Transform, discrete Fourier Transform. Description of numerical filters, classification of numerical filters: finite and infinite impulse response filters (FIR and IIR), analysis of numerical filters, stability of numerical filters, temporal and frequency responses of numerical filters, synthesis of numerical RIF and RII filters.

- Advanced numerical methods and Object Oriented Programming: Java (6 ECTS)

Introduction to the finite differences methods, application to 1D heat transfert equation and 1D diffusion equation. Introduction to the finite element method, application to the electrostatic sensor and Joule heating. Basic concepts of object-oriented programming: objects, classes, methods, constructors, inheritance, overloading and redefinition, encapsulation. Packages of basic and graphics toolkit (Swing classes). Management of events. Applets and the Internet. Threads and synchronization.

- Physics of sensors and Microcontroller and applications (6 ECTS)

Fundamentals of sensors: common features, appropriate readout electronics, economical criteria, examples of complete sensor systems, etc.

Temperature sensors (thermocouples, metal-based resistance thermometers, thermistors) as well as humidity sensors (psychrometer...).

Sensors whose response relates to mechanics: displacement, speed, force, acceleration, fluid pressure, etc.

Radiation sensors (photo-detectors, bolometers, pyroelectric detectors) and magnetic sensors (e.g. Hall effect probe). Electrochemical and biological sensors.

Microcontroller: design and drivers, Programming by interrupt, example in motor control. DSP: design, signal processing in real time on DSP, example in digital signal processing. Applications: Digital control, Closed-loop systems.

- Dynamic systems modelling and analysis (6 ECTS)

Reminders of kinematics of the mechanical systems. Mechanical transmissions. Application of the General
Theorems of the Mechanics. Equations of the movement by the general theorems. Theorem of d' Alembert and equations of Lagrange. Multipliers of Lagrange for the constrained systems. Orthogonal projection on pseudo-velocities. Analytical and numerical resolution of the equations of the movement. Systems balance and stability analysis.

- Italian language (3 ECTS)

- English language (3 ECTS)

\section{Semester S1 in Brescia}

The courses issued in Brescia during the first semester and attained by the Italian students are:

- Laboratory of industrial measurements (6 ECTS) Metrology and mechanical and thermal measurements techniques for industrial applications and diagnostics.

- Numerical Computing with Matlab (6 ECTS)

Approximation of functions and data. Numerical differentiation and integration. Ordinary differential equations. Nonlinear equations. Linear systems. Programming in MATLAB ${ }^{\circledR}$

- Economics applied to Engineering (6 ECTS)

Accounting report, balance worksheet, profit and loss account. Management control system, cost analysis, opportunity cost. Investment assessment.

- Industrial plants (9 ECTS)

General technical and economic issues. Recurrent problems in plants design. Electrical power distribution. Industrial air conditioning design. Industrial lighting design. Fire protection plants and equipment. Inventory management and production planning and scheduling. Job design, work organization and human resources management. Maintenance management.

\section{Semester S2 in Brescia}

Both French and Italian students attend the following courses, taught partly in Italian and partly in English:

- Servosystems and robotics (9 ECTS)

Part 1: methodologies for kinematics and dynamics modeling, basic control algorithms, trajectories generation.

Part 2: choice or design of robots and their adaptation to different applications, their insertion in working cells, development of mathematical models for simulation and control. Main European technical standards.

- Control Systems Technology (9 ECTS)

Industrial control systems. PID controllers. Control architectures. Predictive control. Fuzzy control.

- Microprocessor Instrumentation (9 ECTS)

Microprocessor system and its interfaces. Central Processing Unit (CPU). Programming a microprocessor. Communication systems and interfaces. Digital instrumentation. Laboratory of digital instrumentation.

- Biomechanics (3 ECTS)

Measure, analysis and simulation of the human movement (kinematics, statics, and dynamics). Anthropo- 
metry. The musculoskeletal system. Applications to daily and sport motion. Bases of passive and active prosthesis.

\section{Semester S3 in Paris}

Both French and Italian students together with other foreign students attend the following courses, taught in English:

- Human verbal analysis (3 ECTS)

Introduction to interaction and to speech processing (signal and phonetic). Speech representations (Acoustical, Phonetic, Prosodic, Semantic). Psycho-acoustic. Algorithmic aspects for speaker states classification. Application to speech assessment and Human-Robot Interaction.

- Haptics and applications in teleoperation and virtual reality (3 ECTS)

Method of synthesis and object mechanical properties and their applications to virtual reality systems. Force feedback control in teleoperation. Master-slave control. Methods and performances. Force feedback in haptic interfaces and the transparency challenge.

- Models of sensori-motor functions and learning (3 ECTS)

The organization of movement: issues, observations, concepts and models. Mathematics of control. Motor control: anatomo-physiological organization of motor control (muscles, reflexes and circuits), computational motor control (dynamical systems, internal models, optimality), models of motor control (muscle models, equilibrium point and impedance control, optimal control). Motor Learning: introduction (motor adaptation, skill acquisition, action selection, reinforcement learning), reinforcement learning tools and supervised learning tools for motor Learning.

- Multimodal perception for human pose estimation (3 ECTS)

Sensors for human pose estimation: force sensors, accelerometers, EMG, motion capture. Estimation theory: bias, covariance, efficiency, asymptotic properties, classical estimators (least square, maximum likelihood, etc.) and state estimators for stochastic systems: Kalman filter, extended Kalman filter, unscented Kalman filter, particle filter, etc. Sensors fusion.

- Programming real-time systems (3 ECTS)

Introduction to real-time operating systems, tasks and kernel objects (semaphores, message queues, etc), scheduling, synchronization and communication. Timer services, I/O subsystem, exceptions and interrupts.

- Physically interacting systems and their applications to rehabilitation (3 ECTS)

State-of-the-art in rehabilitation robotics including medical aspects such as protocol definition, pathology description, motor learning fundaments, etc. Kinematics, statics and dynamics aspects of the interactive robot design, all based on elementary biomechanics, as well as the most popular actuation solutions. Problems and solutions encountered in force control of robots, in a conventional way. Current tendencies of anthropocentric robot control, i.e. robot control methods explicitly accounting for human being models in their controller.

- Advanced robotics (3 ECTS)

Kinematic description and parametric representation of systems. Holonome and non-holonome constraints. Homogeneous transformations. Direct and inverse geometrical model by analytical, numeric methods and by methods of homotopy. Laws of transmission of movements in the simple and complex systems. Symbolic methods of elimination for the obtaining of the input-output relationships. Singularities. Resolution of the kinematic problems. Resolution of the inverse kinematic problems - Forced systems and redundant systems. Dynamic model by the general theorems and the equations of the movement by the equations of Lagrange. Generation of articular and Cartesian trajectories.

- Safety, evaluation and acceptability aspects in rehabilitation and assistive technologies (3 ECTS)

Definition and application of basic concepts for risk management: risk, harm, safety, hazard, hazardous situation, safety integrity, safety integrity level, etc. Risk analysis methods (Fault Tree Analysis, Failure Mode Effects and Criticality Analysis, HAZOP, etc.) based on system modelling. Risk reduction techniques for medical robots (Hardware and software fault avoidance techniques, fault tolerance mechanisms, etc.). Certification principles and regulations. Acceptability and ergonomy of the assistive technologies. Relationship between Assistive Technology and Inclusive Design. Clinical trials methodology and protocol design. Engineers, designers and technicians collaboration methodology.

- Image processing for human analysis (3 ECTS) Presentation of images. Images filtering. Mathematical Morphology. Edge detection. Human detection with background subtraction. Motion estimation. Basis of tracking

- Free unit (3 ECTS) 